\title{
Development of transient expression assay for Cannabis sativa which revealed differential Agrobacterium susceptibility among cannabis cultivars
}

\author{
Alexei Sorokin", Narendra Singh Yadav", Daniel Gaudet, Igor Kovalchuk* \\ Department of Biological Sciences, University of Lethbridge, Lethbridge T1K 3M4, Alberta, \\ Canada \\ \# These authors have contributed equally to this work. \\ *Corresponding author: igor.kovalchuk@uleth.ca
}

\begin{abstract}
In plant biology, transient expression analysis plays a vital role to provide a fast method to study the gene of interest and subsequently leads the path to develop an improved crop variety with better agronomic traits. In this study, we have reported a rapid and efficient method for transient expression in Cannabis sativa seedlings using Agrobacterium tumefaciens-mediated transformation. A. tumefaciens strain EHA105 carrying the pCAMBIA1301 construct with uidA gene was used to transform cannabis seedlings and the GUS assay was used to detect the uidA expression. A $1 \%$ hydrogen peroxide $\left(\mathrm{H}_{2} \mathrm{O}_{2}\right)$ solution was used for both seed sterilization and rapid germination steps. Transient transformation revealed that both cotyledons and young true leaves are amenable to transformation. Comparison to Nicotiana tabacum (tobacco) showed that cannabis seedlings were less susceptible to transformation with Agrobacterium tumefaciens. The susceptibility to Agrobacterium infection also varied with the different cannabis cultivars. The
\end{abstract}


method established in this study has potential to be an important tool for gene-function studies and genetic improvement in cannabis.

Keywords: Cannabis sativa, Rapid germination, Nicotiana Benthamiana, Agrobacteriummediated transformation, transient expression, GUS assay.

\section{Introduction}

Cannabis sativa is an annual dioecious herb that belongs to family Cannabaceae. Historically, cannabis has been widely cultivated as a source of seed oil, fiber and intoxicating resin. First written evidence of using cannabis in medicinal practices is described in the compendium of Chinese medicinal herbs by Emperor Shen Nung, dated 2737 B.C.E. [1]. In the last decades, the therapeutic potential of cannabinoids has been reported for the treatment of a range of human diseases from complex neurological diseases to cancer [2]. Although cannabis is best known for the psychoactive compound D9-tetrahydrocannabinol (THC), it also contains varying levels of non-psychoactive cannabinoids such as cannabidiol (CBD), cannabigerol (CBG), D9tetrahydrocannabivarin $(\mathrm{THCV})$, and cannabichromene $(\mathrm{CBC})$, that show promising therapeutic properties and in some cases mitigate the psychoactive effects of THC [3].

Considering the enormous economic importance, it is worthy to study the functional genomics of cannabis. The transient expression analysis is an important tool for functional genomics study. Agrobacterium-mediated transformation is commonly used to achieve both transient and stable gene expression in plants. Wahby et al. [4] reported that $C$. sativa hypocotyl tissues exhibited high susceptibility to Agrobacterium infection/transformation than other tissues. Recently, Chaohua et al. [5] established regeneration protocol that uses cotyledons of $C$. sativa as an explant. Considering aforementioned reports, we decided to use intact cannabis seedlings for establishment 
of transient expression protocol. Such protocol can be used for functional genomics study and for the development of stable transformation protocol. In this study, we have reported an efficient and reproducible method for transient expression analysis in C. sativa seedlings using Agrobacterium tumefaciens-mediated transformation and demonstrated that cannabis is less susceptible to Agrobacterium transformation than tobacco. Further, we also displayed that susceptibility to Agrobacterium infection also varied with the different cannabis cultivars.

\section{Materials and Methods}

\section{Materials}

\section{A. Biological materials}

1. Agrobacterium tumefaciens strain (EHA105) carrying binary vector pCAMBIA1301 with uidA gene.

2. Cannabis sativa (Candida CD-1, Night in Gale, Green Crack CBD, and Holy Grail x CD-1 cultivars) and Nicotiana benthamiana seeds.

\section{B. Chemicals}

1. Hydrogen Peroxide $30 \%$ (Merck ${ }^{\circledR}$, catalog number: 1072091000)

2. Agrobacterium liquid growth medium (YEP liquid medium) (see Recipes)

3. Agrobacterium liquid induction medium (see Recipes)

4. Histochemical GUS staining solution (see Recipes)

5. MS solid media (see Recipes)

6. $\mathrm{MgSO} 4$ (Sigma-Aldrich, catalog number: MX0075-1)

7. Acetosyringone (Sigma-Aldrich, catalog number: D134406)

8. Murashige \& Skoog Basal Medium with Vitamins (PhytoTechnology Laboratories ${ }^{\circledR}$, catalog number: M519) 
9. Kanamycin sulfate (PhytoTechnology Laboratories ${ }^{\circledR}$, catalog number: K378)

10. Rifampicin (Sigma-Aldrich, catalog number: R3501)

11. Selective antibiotics: Kanamycin, Rifampicin

12. $70 \%$ Ethanol

13. Sucrose (Sigma-Aldrich, catalog number: S0389)

14. MES (Sigma-Aldrich, catalog number: M3671)

15. Agar

16. Yeast extract

17. $\mathrm{NaCl}$

18. Peptone

19. EDTA ( $\mathrm{pH} 8.0$ ) (Sigma-Aldrich, catalog number: E9884)

20. Sodium phosphate buffer ( $\mathrm{pH} 7.0)$

21. Triton X-100 (Sigma-Aldrich, catalog number: 234729)

22. Potassium ferricyanide (Sigma-Aldrich, catalog number: 702587)

23. Potassium ferrocyanide (Sigma-Aldrich, catalog number: P3289)

24. X-Gluc (Sigma-Aldrich, catalog number: R0852)

\section{Plasticware}

1. Sterile empty 100 x 15 mm Petri plates (VWR International, catalog number: 25384-

2. Sterile disposable $50 \mathrm{ml}$ screw-cap centrifuge tubes $\left(\mathrm{BD}\right.$, Falcon $^{\mathrm{TM}}$, catalog number: 352070)

3. Plastic pipette tips $(20,200$, and $1,000 \mu \mathrm{l})$

4. Disposable Cuvettes 
5. Sterile filter papers

\section{Equipment}

1. Spectrophotometer

2. Allegra Benchtop Centrifuge X-12 (Beckman Coulter)

3. Micro-centrifuge

4. Laminar flow hood

5. Eppendorf Research ${ }^{\circledR}$ plus 10, 20, 200, and 1,000 $\mu 1$

6. Analytical balance

7. Top loading electronic balance

8. $\mathrm{pH}$ meter

9. Vortex mixer

10. Freezer $\left(-80^{\circ} \mathrm{C}\right)$ (e.g. New Brunswick, model:)

11. Sterile forceps and scalpel (sterilized by heat treatment using a Bunsen burner)

12. Sterile inoculating loop

13. A desiccator attached to a vacuum pump (Brinkman DistiVac)

14.

15. Growth chamber

16. Shaker incubator $\left(28^{\circ} \mathrm{C}, 220 \mathrm{rpm}\right)$

17. Incubator $37^{\circ} \mathrm{C}$

18. Fluorescent microscope (Zeiss Observer Z1)

\section{Methods}

\section{A. Germination and seedlings development (performed under sterile conditions)}


1. For germination, seeds were soaked in a $1 \%$ hydrogen peroxide solution incubated overnight for $24 \mathrm{hrs}$ at room temperature in the dark. The following day, radicles with hypocotyl are visible (Figure 1).

2. Transfer germinated seeds into fresh $1 \% \mathrm{H}_{2} \mathrm{O}_{2}$ solution and further incubate for 3-4 days until cotyledons have fully opened and two early true leaves are visible.

3. Remove remaining seed coats using sterile scalpel and forceps.

4. Sterilize seedlings without seed coats by soaking them in $1 \%$ hydrogen peroxide for $5 \mathrm{~min}$.

5. Prior to transformation rinse seedlings in sterile water 3 times to remove remaining hydrogen peroxide.

\section{B. Preparation of Agrobacterium cells culture (all steps performed under sterile conditions)}

1. Two days before transformation, inoculate $100 \mathrm{ml}$ of YEP (containing $50 \mu \mathrm{g} / \mathrm{mL}$ Kanamycin and $25 \mu \mathrm{g} / \mathrm{mL}$ Rifampicin) with Agrobacterium from glycerol stock and culture at $28^{\circ} \mathrm{C}$ in an incubator shaker $220 \mathrm{rpm}$ overnight.

2. Next day centrifuge the Agrobacterium cells culture at $4,000 \mathrm{x}$ g for $15 \mathrm{~min}$ at RT.

3. Remove supernatant and add $3 \mathrm{ml}$ of $10 \mathrm{mM} \mathrm{MgSO} 4$, resuspend the Agrobacterium pellet.

4. Repeat steps 2 and 3.

5. Centrifuge a third time, remove supernatant.

6. Resuspend the Agrobacterium pellet in an appropriate volume of induction medium (MS liquid media) so that the final OD600 $=0.6$.

7. Add $100 \mathrm{mM}$ acetosyringone to final concentration $100 \mu \mathrm{M}$.

\section{Co-cultivation (all steps performed under sterile conditions)}


1. Place sterilized seedlings in $50 \mathrm{ml}$ Falcon tubes with $30 \mathrm{ml}$ of the Agrobacterium cells suspension (Agrobacterium cells in induction medium supplemented with acetosyringone).

2. Place the tubes into a sterile vacuum chamber and apply vacuum for 10-20 min.

3. Transfer seedlings to a sterile filter paper to remove the excess Agrobacterium cell culture.

4. Transfer the seedlings to $90 \mathrm{~mm}$ petri dishes containing MS media (10 seedlings per plate). Spread them evenly on the plate using forceps. Seal the Petri dishes with parafilm.

5. Co-cultivate the seedlings and the Agrobacterium cells for three days in the dark at $25^{\circ} \mathrm{C}$.

6. After co-cultivation, seedlings can be used directly for GUS staining or can be frozen at $-80^{\circ} \mathrm{C}$ for further analysis e.g. MUG assay, PCR analysis.

\section{Transient expression analysis by GUS assay}

1. After 3-days co-cultivation, rinse seedlings in sterile water.

2. Place seedlings in $50 \mathrm{ml}$ Falcon tubes with Histochemical GUS staining solution.

3. Apply vacuum for $10 \mathrm{~min}$.

4. Incubate overnight at $37^{\circ} \mathrm{C}$.

5. After staining, rinse seedlings in $70 \%$ ethanol to remove excessive stain.

6. Keep seedlings in $70 \%$ alcohol for distaining of chlorophyll.

\section{Recipes}

1. YEP liquid medium (1L)

$10 \mathrm{~g}$ Yeast extract 
$10 \mathrm{~g}$ Peptone

$5 \mathrm{~g} \mathrm{NaCl}$

pH 7.0 Autoclave

2. GM medium (1L)

4.43 g Murashige \& Skoog Basal Medium with Vitamins

$10 \mathrm{~g}$ Sucrose

$500 \mathrm{mg}$ MES

pH 5.7, autoclave

3. MS sold media (1L)

4.43 g Murashige \& Skoog Basal Medium with Vitamins

8 g Agar

pH 5.7, autoclave

4. Histochemical GUS stain solution

$2 \mathrm{mM}$ Potassium ferrocyanide

$2 \mathrm{mM}$ Potassium ferricyanide

100 mM Sodium Phosphate Buffer

$500 \mathrm{mg}$ X-Gluc (pre dissolve in dimethyl formamide)

$0.1 \%$ Triton $X-100$

$1 \mathrm{mM}$ EDTA

\section{Results and Discussion}

Transient expression analysis provides a rapid method to study the function of genes. Transient transformation protocols may also be used to develop stable transformation protocols. In this study, we have reported a rapid and efficient method for transient expression in Cannabis sativa seedlings 
using Agrobacterium tumefaciens-mediated transformation. The Agrobacterium tumefaciens strain EHA105 carrying the pCAMBIA1301 construct was used to transform cannabis seedlings and the GUS assay was used to detect the transgenics.

Hydrogen peroxide $\left(\mathrm{H}_{2} \mathrm{O}_{2}\right)$ has been used as a disinfectant for seeds for decades [6]. The 1\% $\mathrm{H}_{2} \mathrm{O}_{2}$ was used for both seed sterilization and rapid germination step in solution (unpublished protocol). This presents significant advantage over mercuric chloride or bleach that require additional washing of seeds and germination step in MS medium. This is a very rapid germination method as germination occurs in $24 \mathrm{~h}$ and seedlings development in $72 \mathrm{~h}$. After 3-4 days of incubation in $1 \% \mathrm{H}_{2} \mathrm{O}_{2}$ solution, seedlings leave seed coats with two fully opened cotyledons and two immature true leaves (unpublished protocol; Fig. 1); seedlings at this developmental stage were used for transformation. Literature survey displayed that different cultivars of cannabis showed different germination response and revealed optimal germination within 4-7 days by using various germination methods [7] and develop a seedling in 5-15 days or even more. Similarly, Çavusoglu and Kabar [8] showed that exogenous application of $\mathrm{H}_{2} \mathrm{O}_{2}$ to seeds of different plant species increases seed germination rates, coleoptile emergence percentages, radicle and coleoptile elongation, and fresh weights of the seedlings.

Figure 1: Various germination development stages of cannabis seedlings in $1 \%$ hydrogen peroxide solution. (a) 24 hours, cannabis embryo absorbs water until radicle breaks through the seed coat. (b) 36 hours, radicle further development and appearance of hypocotyl. (c) 48 hours, cotyledons emerge. (d) 60 hours, seedling almost leave the seed coat. (e) 72 hours, two fully opened cotyledons and two early true leaves are visible.

The overall workflow for the transient transformation is presented in Figure 2. We have used the intact seedlings (two cotyledons stage and two cotyledons with young true leaves stage) for transformation. To enhance the efficiency, we have used the vacuum infiltration followed by 3days co-cultivation on MS media. Vacuum infiltration has been shown to enhance the 
transformation efficiency of Artemisia annua seedlings [9]. To detect the gene transformation in cotyledons and true leaves, the GUS activity assay has been done (Fig. 3). GUS analysis revealed that both cotyledons and young true leaves are amenable to transformation. We have carried out the transformation experiment four times. In one independent experiment, we have used around 30 seedlings and out of 30 seedlings at least 20 seedlings showed the GUS activity spots. Previously, Feeney and Punja [10] successfully demonstrated transient transformation of Cannabis sativa cell suspension cultures with $A$. tumefaciens strain EHA101 carrying the binary vector pNOV3635 with a gene encoding phosphomannose isomerase, although they failed to regenerate fully transgenic cannabis plants. Wahby et al. [4] reported that hypocotyls tissues were most susceptible to $A$. rhizogenes infection, while young leaves and cotyledons did not, even when the bacteria were stimulated with acetosyringone. These contradicting results may be due to different Agrobacterium strains or different cannabis cultivars used in studies.

Figure 2: Workflow for Agrobacterium-mediated transient transformation of cannabis seedlings. Step 1. Sterilization and germination, seeds are soaked in $1 \% \mathrm{H}_{2} \mathrm{O}_{2}$ solution for 24 hours until germination and then transferred into fresh solution. Seeds are then incubated in $1 \% \mathrm{H}_{2} \mathrm{O}_{2}$ until both cotyledons and epicotyl are visible. Step 2. Co-cultivation, vacuum applied to seedlings submerged in Agrobacterium cell suspension, seedlings are then transferred to MS media plates and incubated for three days in complete dark at $25^{\circ} \mathrm{C}$. Step 3. Confirmation of transformation, histochemical GUS assay using transformed seedlings.

Figure 3: Representative images of GUS activity analysis in cotyledons and leaves tissues of cannabis seedlings to confirm the transient transformation. (A) GUS activity analysis in cotyledons (left panel) and true leaves (right panel). (B) Microscopic observation of GUS activity in cotyledons, non-transformed tissue (left panel) and transformed tissue (right panel). Scale bar $100 \mu \mathrm{M}$. (C) Microscopic observation of GUS activity in true leaf, non-transformed tissue (left panel) and transformed tissue (right panel). Scale bar $100 \mu \mathrm{M}$.

Comparative qualitative analysis revealed that cannabis seedlings showed less GUS activity than Nicotiana benthamiana which suggests that cannabis is less susceptible to Agrobacterium infection than Nicotiana benthamiana (Fig. 4A). The susceptibility to Agrobacterium infection also varied with the different cannabis cultivars. The Nightingale cultivar showed higher 
susceptibility than the Green Crack CBD and Holy Grail x CD-1 (Fig. 4B). Previously, Feeney and Punja [11] reported that cannabis is amenable to genetic transformation using Agrobacterium however the plant is recalcitrant to regeneration, impeding the recovery of transgenic cannabis plants.

Figure 4: Representative images of comparative transient expression analysis. (A) Comparative transient expression analysis between cannabis and tobacco. (B) Comparative transient expression analysis between different cannabis cultivars.

In conclusion, we have developed an efficient method for transient expression analysis which has potential to be an important tool for gene-function studies and genetic improvement in $C$. sativa.

\section{Acknowledgements}

We thank NSERC and MITACS for funding our work.

\section{Competing interests}

The authors declare that they have no competing interests.

\section{References}

1. Amar MB. Cannabinoids in medicine: A review of their therapeutic potential. J Ethnopharmacol. 2006;105(1-2): 1-25.

2. MacCallum CA, Russo EB. Practical considerations in medical cannabis administration and dosing. Eur J Intern Med. 2018;49:12-19.

3. Russo EB. Taming THC: potential cannabis synergy and phytocannabinoid-terpenoid entourage effects. Br J Pharmacol. 2011;163(7): 1344-1364.

4. Wahby I, Caba JM, Ligero F. Agrobacterium infection of hemp (Cannabis sativa L.): establishment of hairy root cultures. J Plant Interact. 2013;8(4): 312-320. 
5. Chaohua C, Gonggu Z, Lining Z, Chunsheng G, Qing T, Jianhua C, et al. A rapid shoot regeneration protocol from the cotyledons of hemp (Cannabis sativa L.). Ind Crops Prod. 2016;83: 61-65.

6. Miché L, Balandreau J. Effects of rice seed surface sterilization with hypochlorite on inoculated Burkholderia vietnamiensis. Appl Environ Microbiol. 2001; 67(7): 3046-3052.

7. Wielgus K, Luwanska A, Lassocinski W, Kaczmarek Z. Estimation of Cannabis sativa L. tissue culture conditions essential for callus induction and plant regeneration. J Nat Fibers. (2008);5(3): 199-207.

8. Çavusoglu K, Kabar K. Effects of hydrogen peroxide on the germination and early seedling growth of barley under $\mathrm{NaCl}$ and high temperature stresses. Eurasia J Biosci. 2010; 4(1): 70-79.

9. Ma D, Wang H. Transient Transformation of Artemisia annua. Bioprotocol. 2015;5(10): e1476. doi: 10.21769/BioProtoc.1476.

10. Feeney M, Punja ZK. Tissue culture and Agrobacterium-mediated transformation of hemp (Cannabis sativa L.). In Vitro Cell Dev Biol Plant. 2013;39(6): 578-585.

11. Feeney M, Punja ZK. The role of Agrobacterium-mediated and other gene-transfer technologies in cannabis research and product development. In: Cannabis sativa L.-Botany and Biotechnology. Springer Cham; 2017. pp. 343-363. 


\section{Step 1. Sterilization and germination}

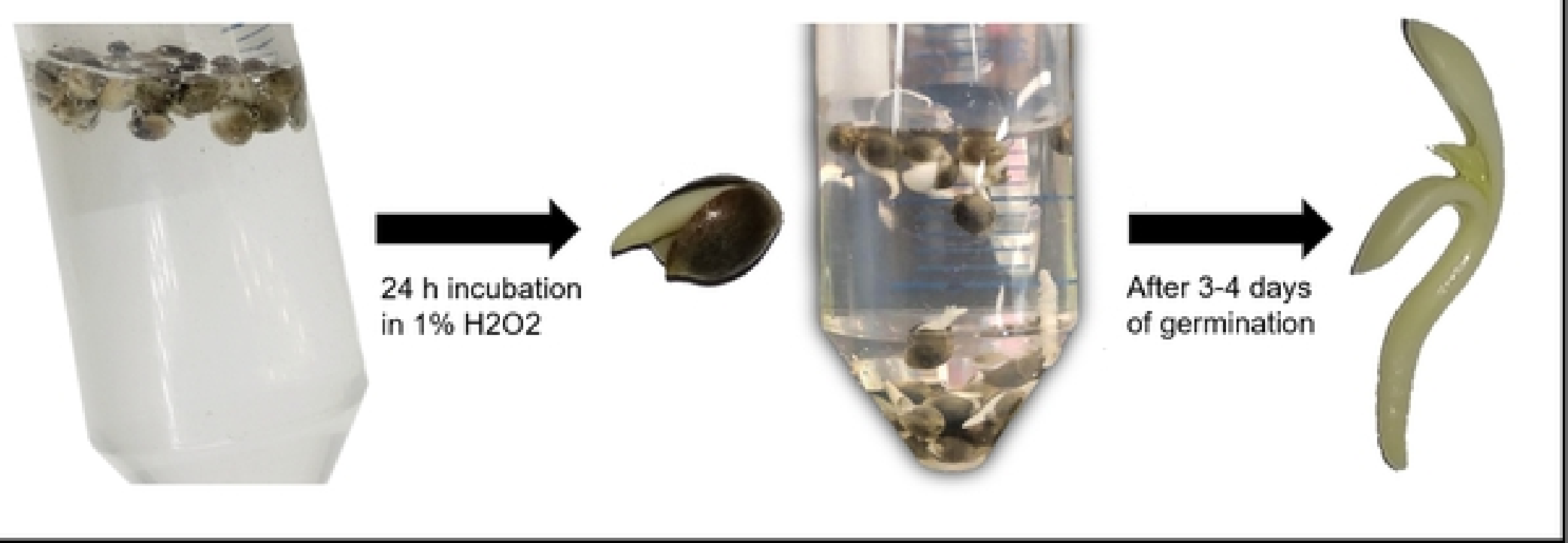

\section{Step 2. Co-cultivation}

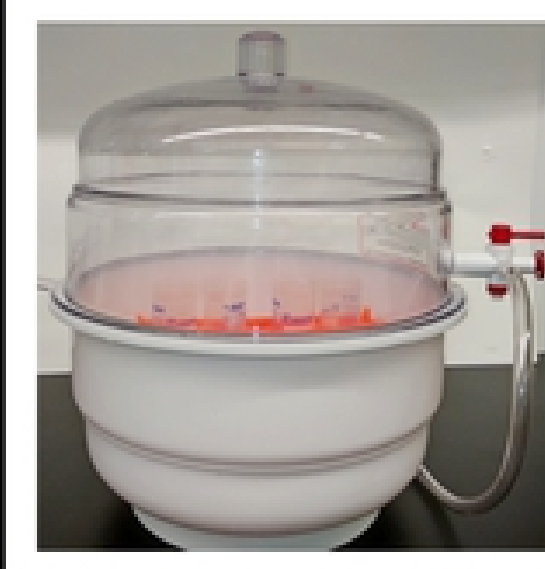

Vacuum infiltration of seedlings in Agrobacterium suspension, 10 minutes, $600 \mathrm{~mm} \mathrm{Hg}$

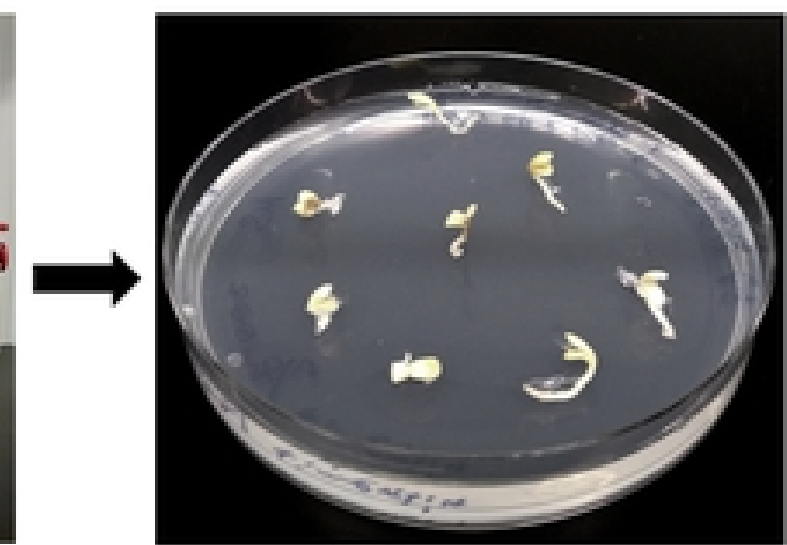

Seedlings after vacuum infiltration transferred to MS media plate

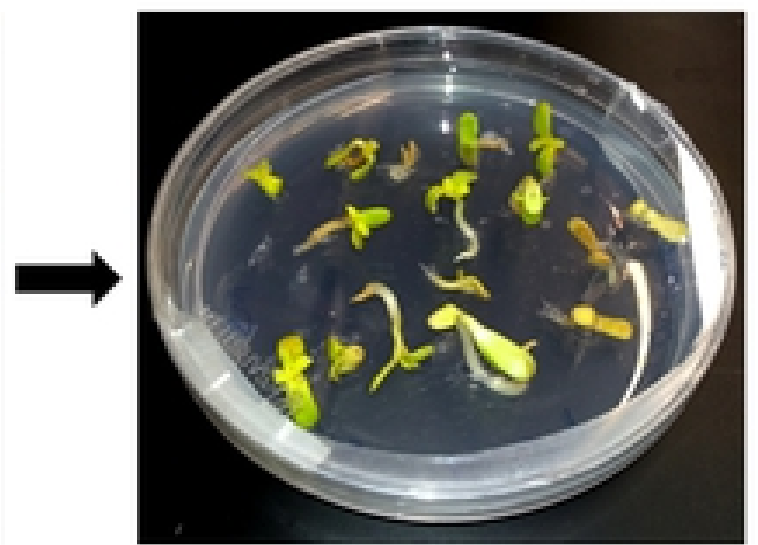

After co-cultivation of the seedlings and the Agrobacterium cells for three days in complete dark at 25
Step 3. Confirmation of transformation

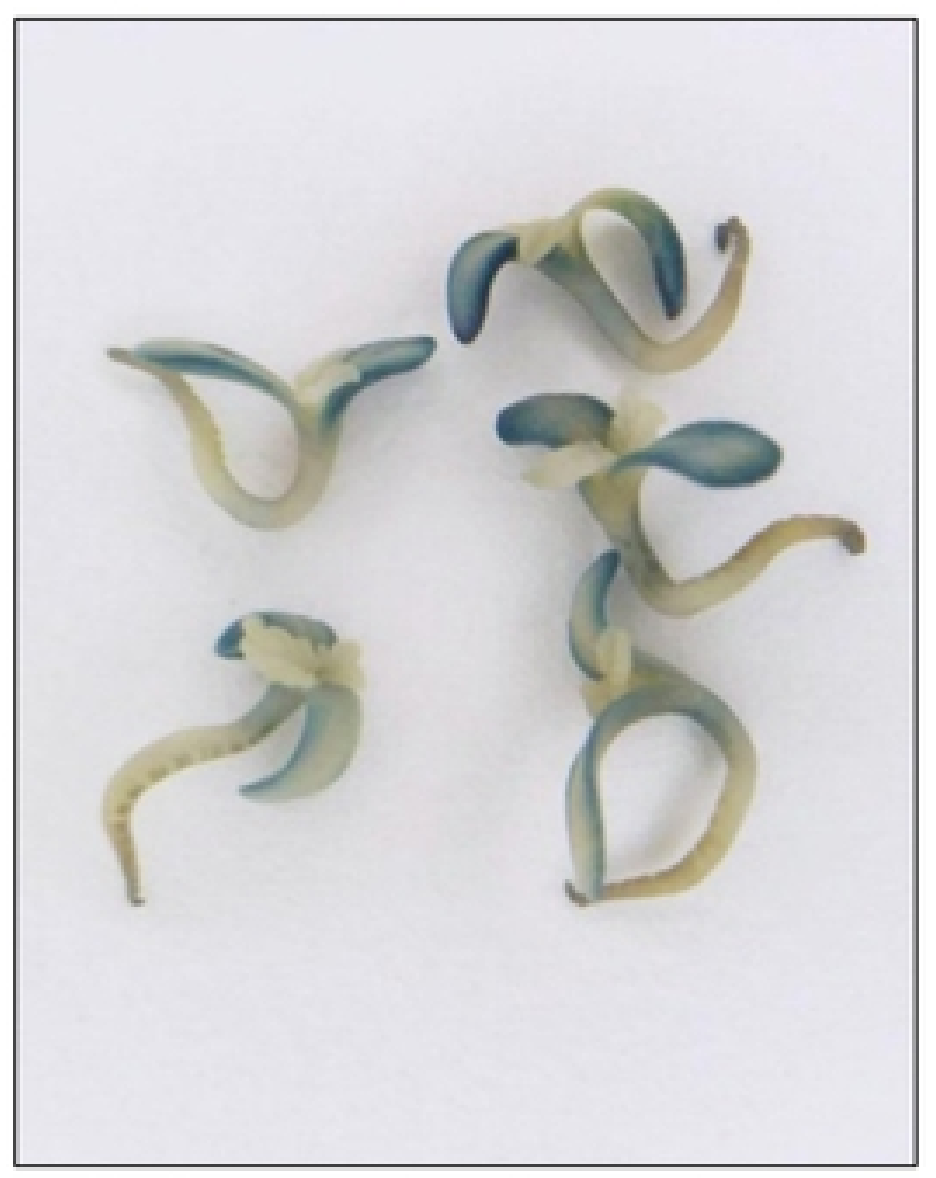

GUS assay for transient expression analysis

Figure 2 
A.

Cotyledons

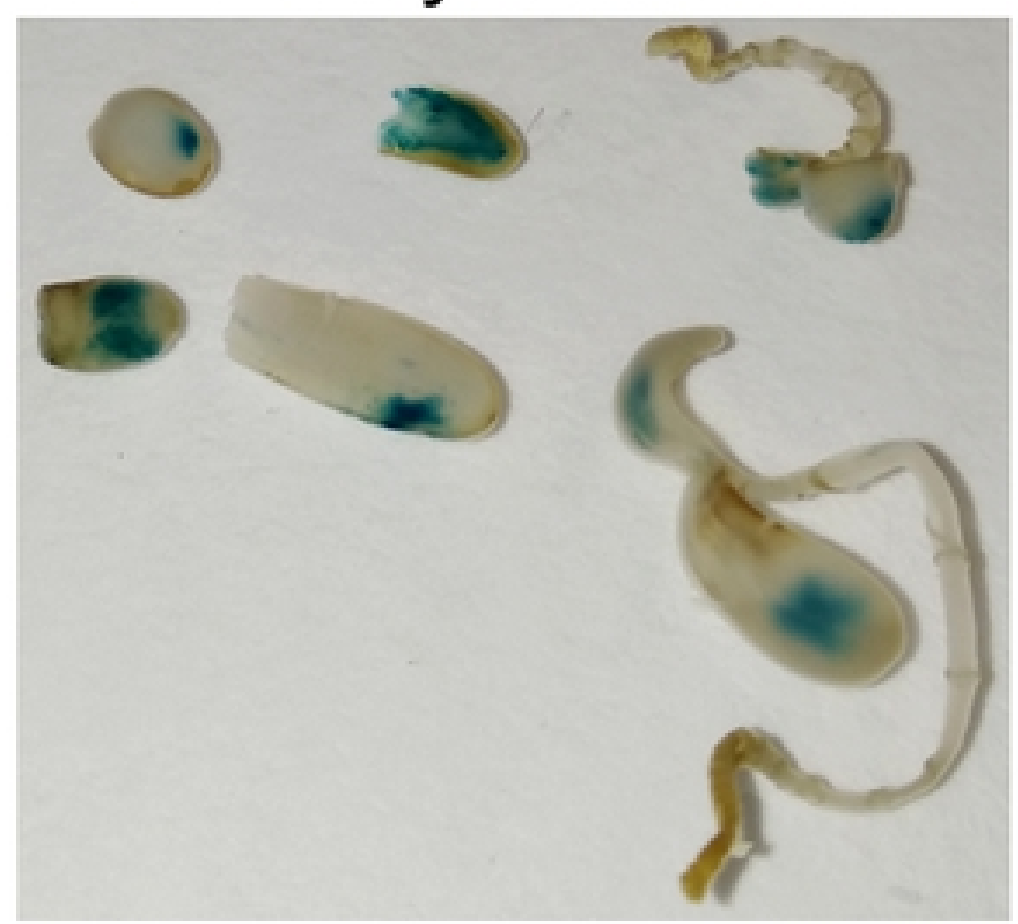

True leaves

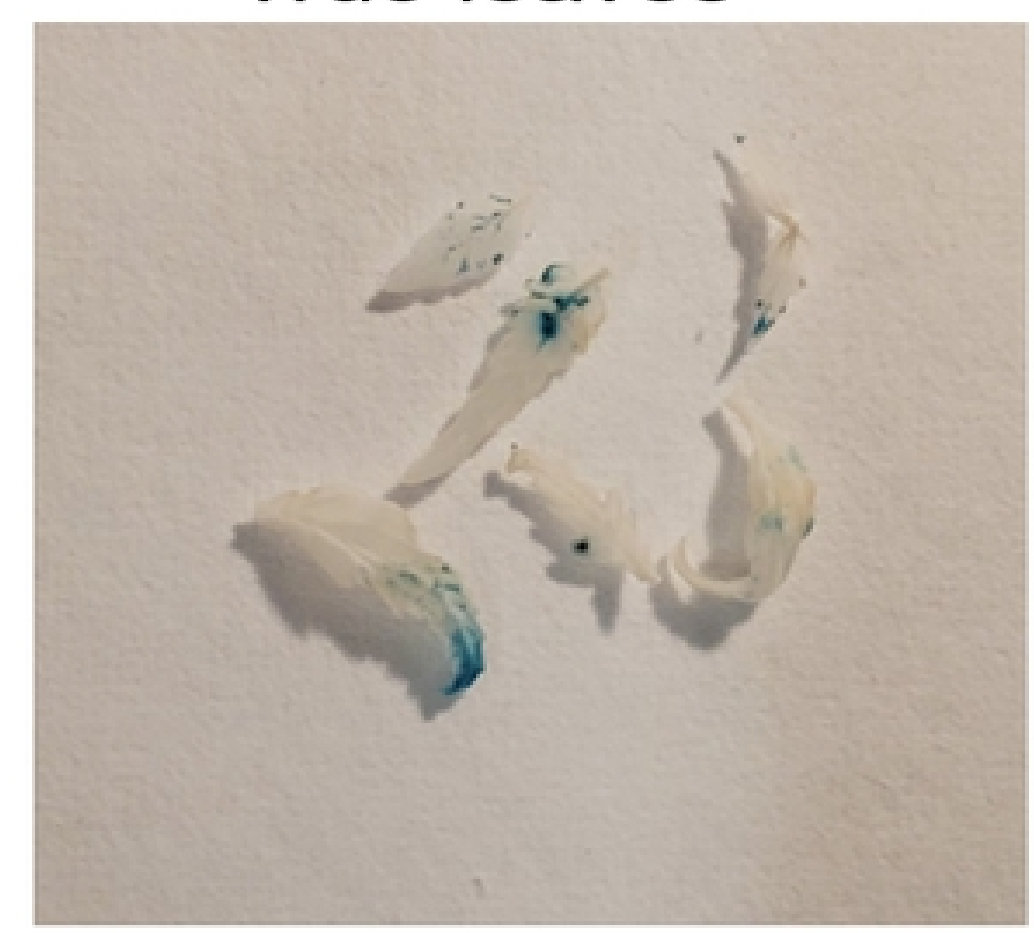

B. Microscopic observation of GUS activity in cotyledons

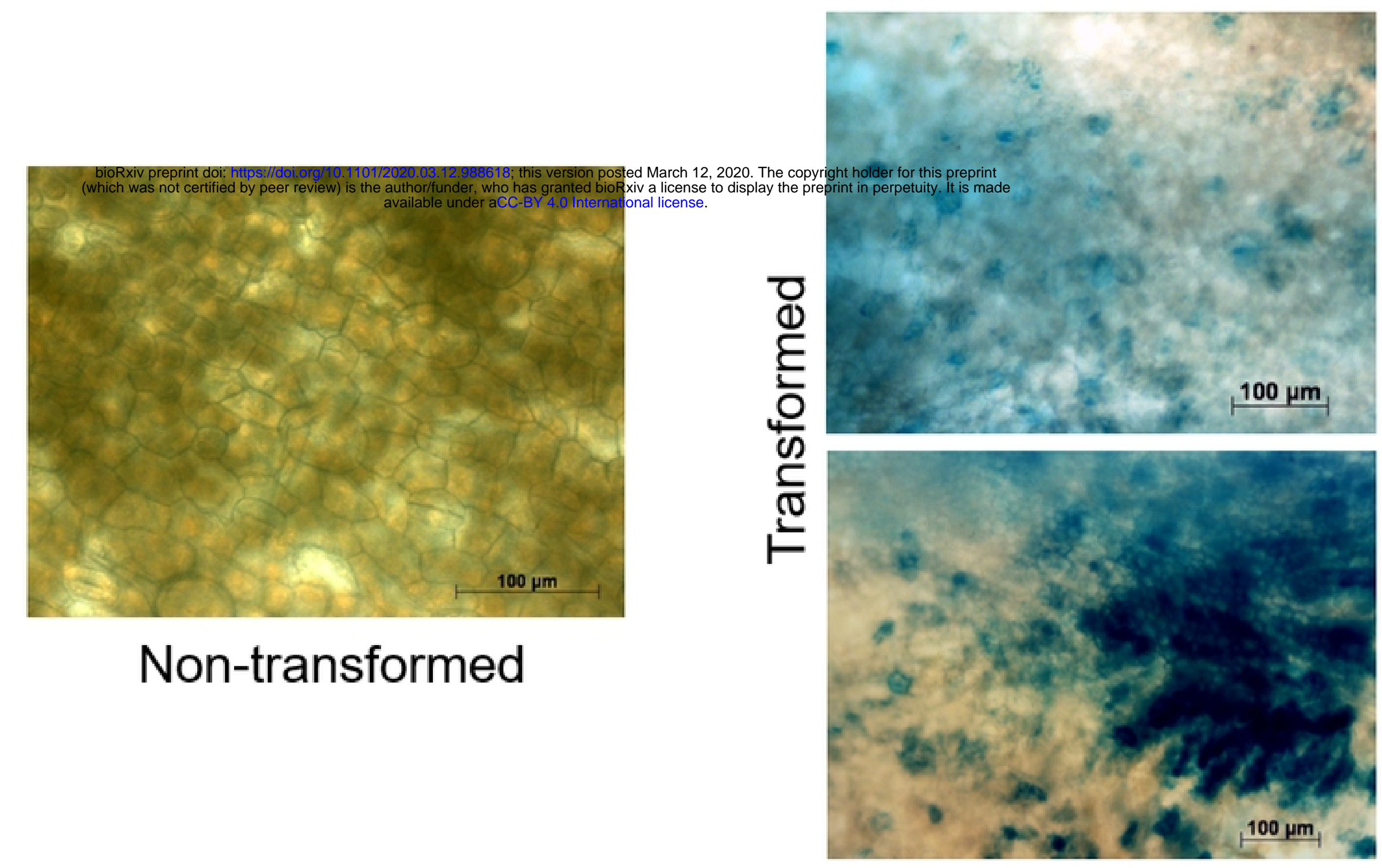

C. Microscopic observation of GUS activity in true leaf

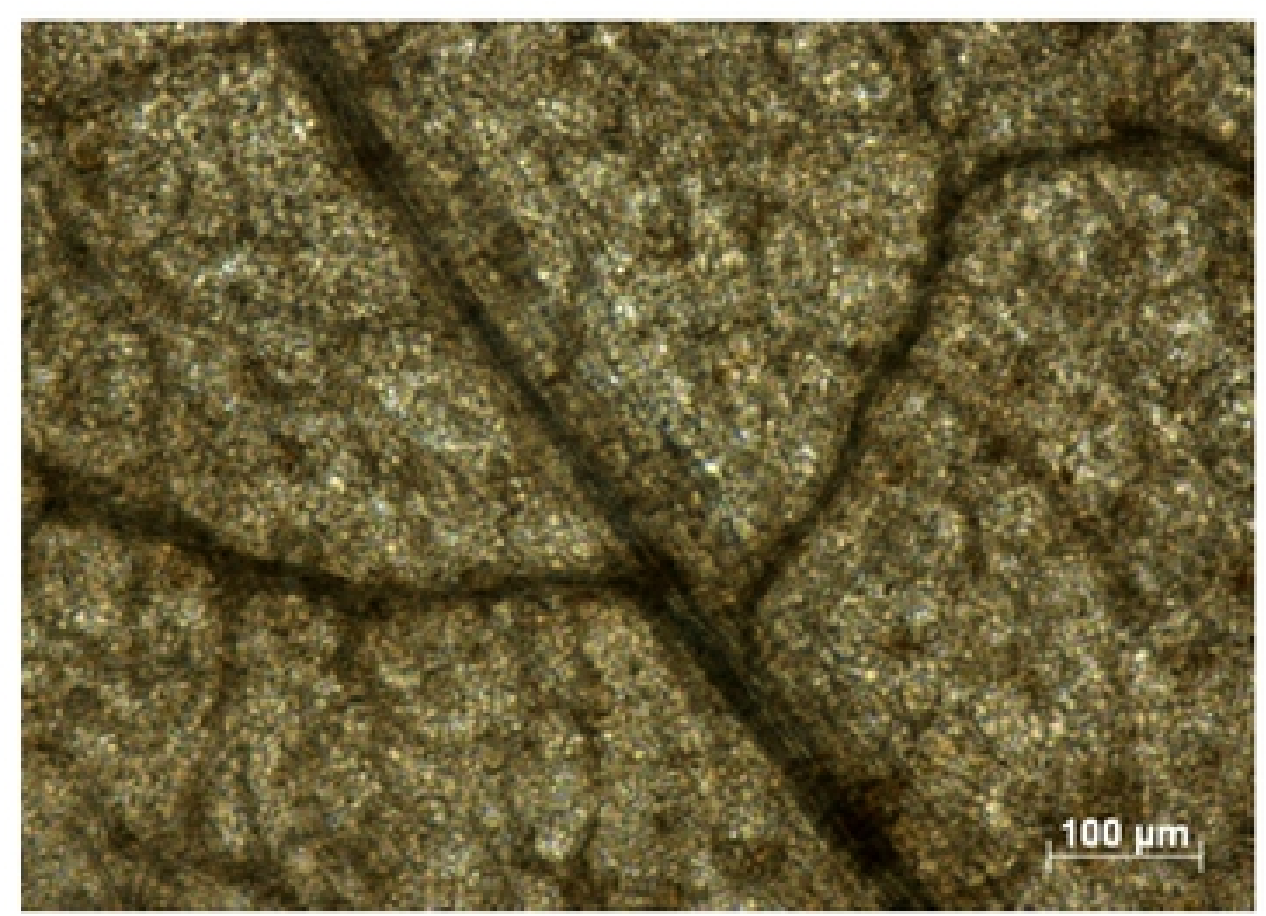

Non-transformed

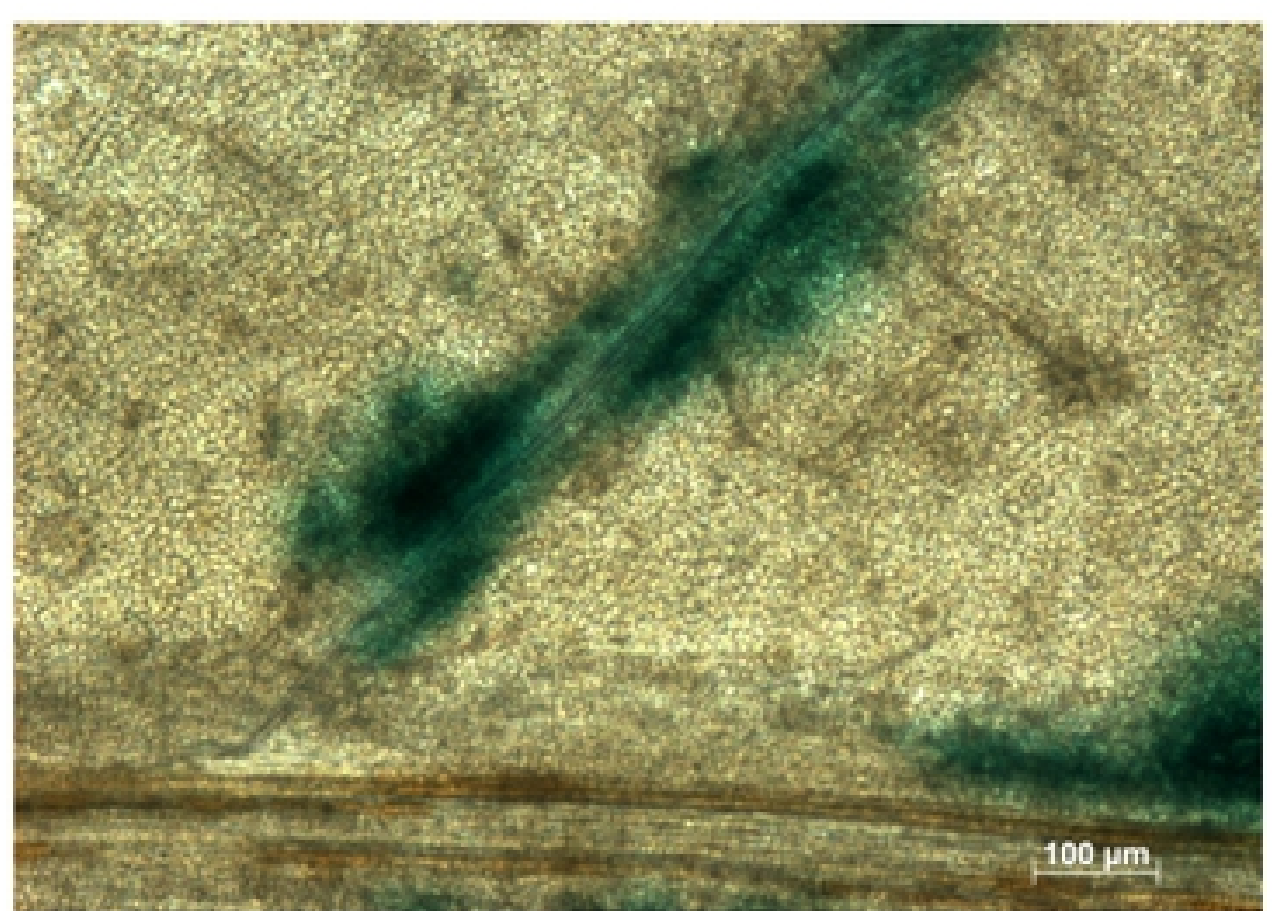

Transformed

Figure 3 
A
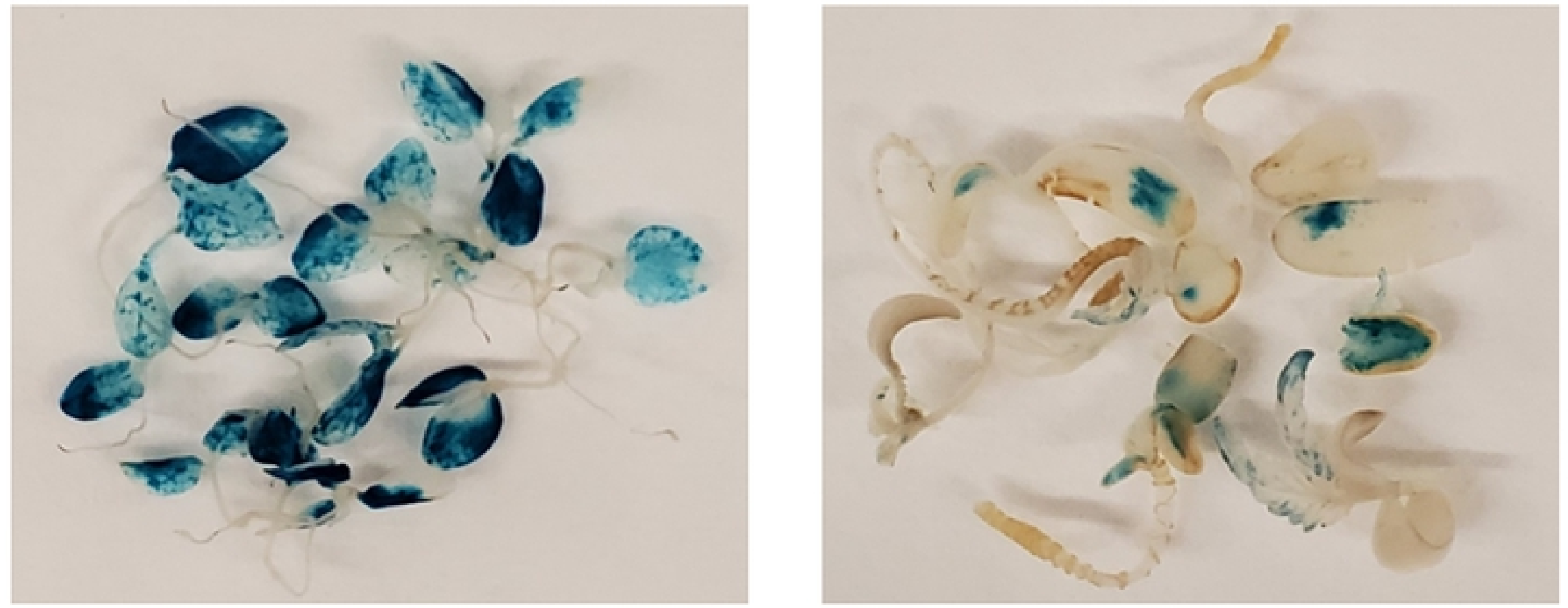

Leaves

\section{Cotyledons}

B

bioRxiv preprint doi: https://doi.org/10.1101/2020.03.12.988618; this version posted March 12, 2020. The copyright holder for this preprint

Nightingale

Green Crack CBD
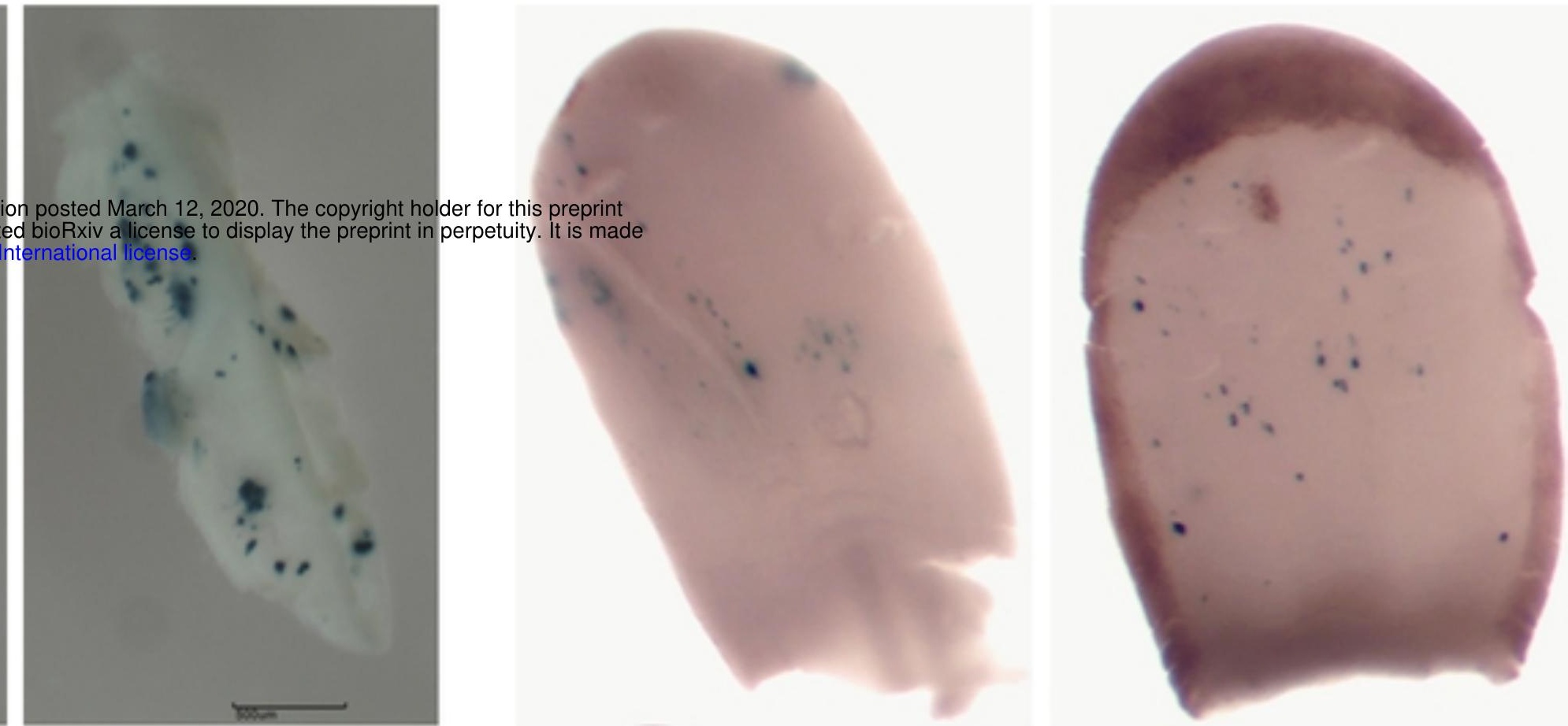

Holy Grail x CD-1

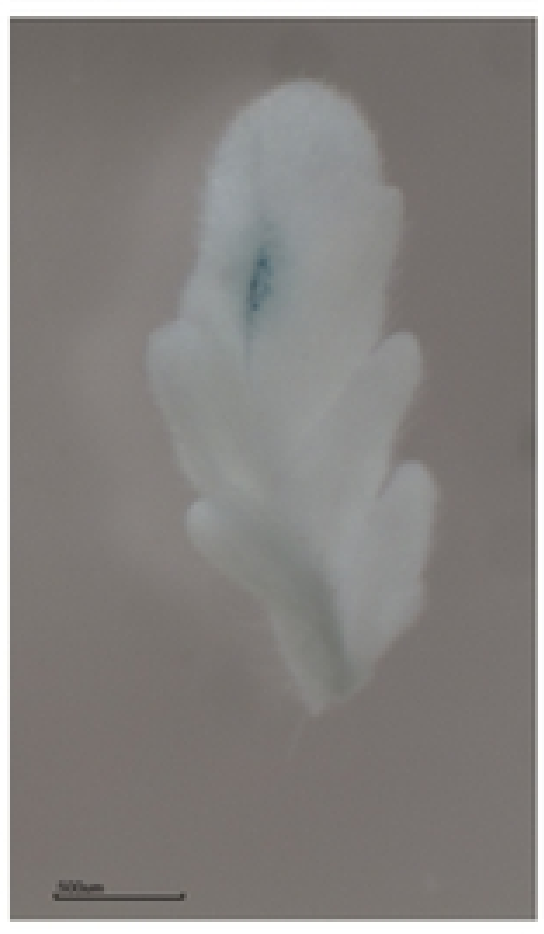

thin
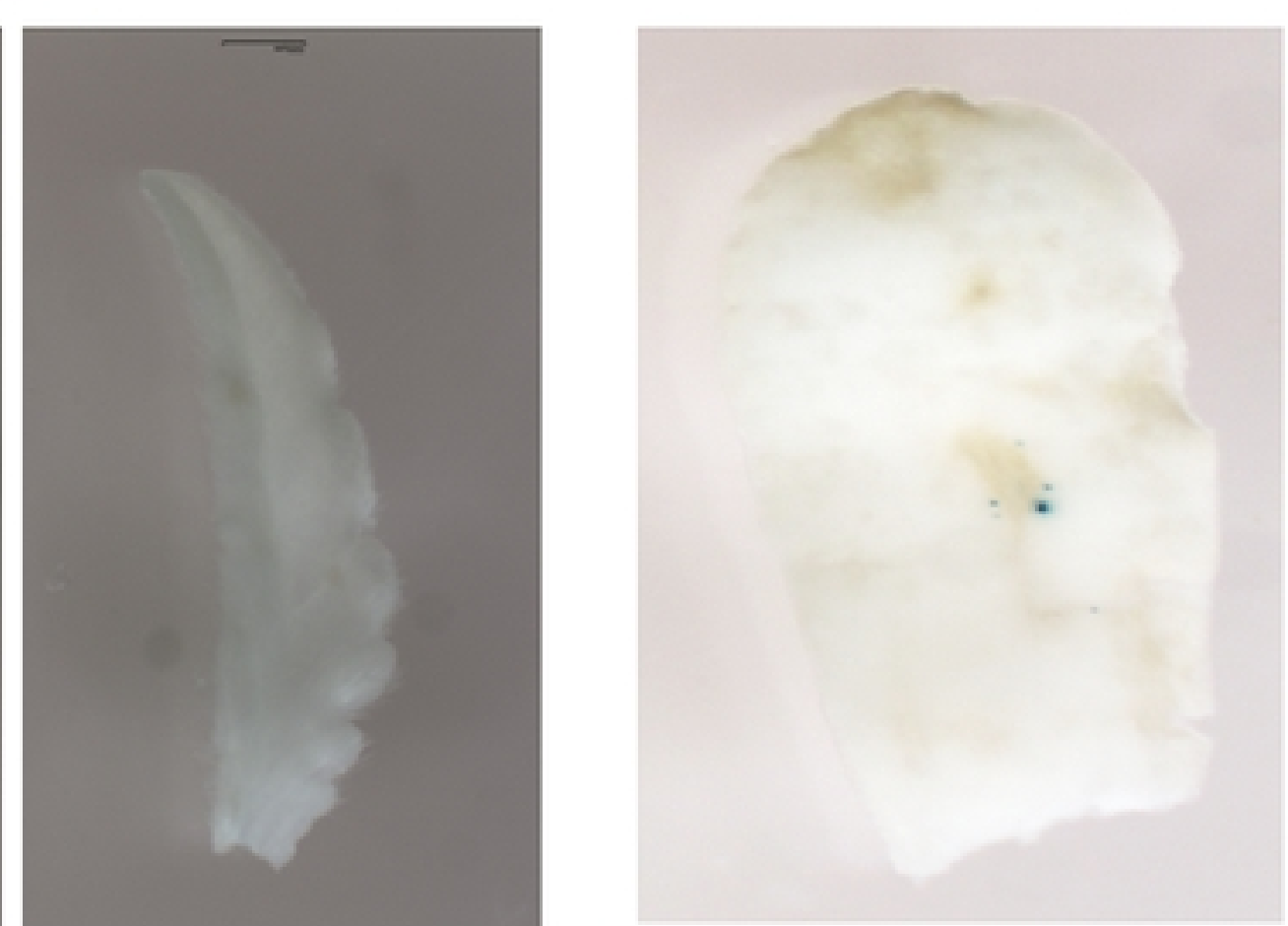

Figure 4 